\title{
Inclusão e qualidade de vida na educação do alunado com deficiência*
}

Miguel Angel Verdugo Alonso

Universidad de Salamanca

Laura Elísabet Gómez Sánchez

Universidad de Valladolid

Alba Rodriguez Aguillela

Instituto Universitario de Integración en la Comunidad

\section{Resumo}

O objetivo deste trabalho consiste em realizar uma revisão teórica e crítica da aplicação dos conceitos de inclusão e qualidade de vida no âmbito da educação de pessoas com deficiência, como agentes de mudança. Para este fim, apresenta-se, resumidamente, a evolução da situação e os princípios educacionais na Espanha nos últimos anos. Em seguida são abordadas evidências sobre os progressos alcançados em matéria de educação inclusiva no país, bem como os paradoxos e os problemas encontrados na sua implementação pelos principais agentes implicados: alunos e alunas com deficiência, familiares e profissionais. Tais evidências ressaltam a necessidade de relançar a aplicação da verdadeira e correta implementação do processo inclusivo. Finalmente, defende-se o modelo de qualidade de vida como agente de mudança e inovação no âmbito escolar.

Palavras-chave: Educação Inclusiva. Qualidade de vida. Deficiência Intelectual. Necessidades Educativas Especiais.

* Tradução: Amaralina Miranda de Souza. 


\section{Inclusion and quality of life in the education of students with disabilities}

The aim of this paper is to present a theoretical and critical review of the concepts of inclusion and quality of life in the ambit of education for people with disabilities. For that purpose, we will start by briefly presenting the evolution of the situation and the educational principles in Spain during the last years. Next, we will provide evidence of the progress in inclusive education in our country, as well as the paradoxes and problems that have been recently found in its implementation by the different social agents involved in this process: students with disabilities, relatives and professionals. This evidence shows the need to relaunch the real and right implementation of the inclusive process. Finally, the "Quality of Life" model is suggested as an agent of change and innovation in the school.

Keywords: Inclusive education. Quality of life. Intellectual disabilities. Special educational needs.

\section{Inclusión y calidad de vida en la educación del alumno con discapacidad}

El objetivo de este artículo consiste en realizar una revisión teórica y crítica acerca de la aplicación de los conceptos de inclusión y calidad de vida en el ámbito educativo de las personas con discapacidad como agentes de cambio. Para ello, se presenta brevemente la evolución de la situación y los principios educativos en España en los últimos años. A continuación, se aportan evidencias acerca de los avances en la inclusión educativa logrados en nuestro país así como las paradojas y problemas encontrados en su implementación por parte de los principales agentes implicados: alumnos y alumnas con discapacidad, familiares y profesionales. Tales evidencias ponen de manifiesto la necesidad de impulsar de nuevo la verdadera y correcta implementación del proceso inclusivo. Finalmente, se plantea el modelo de calidad de vida como agente de cambio e innovación en el ámbito escolar.

Palabras clave: Inclusión educativa. Calidad de vida. Discapacidad intelectual. Necesidades Educativas Especiales. 


\section{Introdução}

Embora desde os anos de 1970 tenha começado a se espalhar pelo mundo a perspectiva integradora na educação - que defende a unificação da educação geral e a educação especial em um único sistema educacional e critica a ineficiência da segregação nas escolas -, esta veio a se concretizar somente em 1985, quando o Ministério da Educação da Espanha se comprometeu a integrar os alunos com deficiência nas escolas públicas de ensino regular e nas escolas subsidiadas através do Real Decreto 334/1985 de 06 de Março de 1985 (Ordenação da Educação Especial). Este decreto se destinava não só aos alunos do ensino básico obrigatório, mas incluiu também a formação profissional, a educação de adultos e o ensino universitário, dando ênfase a aspectos-chave no âmbito da deficiência, tais como os apoios, valorização e orientação educativa, reforço pedagógico e atendimento personalizado. Começa então um processo legal e de aplicação experimental da integração educativa (Verdugo et al., 2009) que continua até hoje com a atual Lei Orgânica 2/2006 da Educação (LOE), com base no princípio da educação inclusiva e com a ratificação da Convenção Internacional da Organização das Nações Unidas sobre os Direitos das Pessoas com Deficiência, em vigor desde 03 maio de 2008 (BOE de 21 de abril de 2008), como parte do ordenamento jurídico espanhol. Apesar das mudanças significativas introduzidas na educação nos últimos anos, especialmente no nível teórico da legislação, a aplicação do conceito de inclusão tem sido caracterizada pelas carências e desigualdades na prática educativa.

\section{Evolução dos princípios educativos}

Os princípios educativos na Espanha, como no resto do mundo, têm evoluído consideravelmente. Desde os tempos caracterizados pela negligência ou abandono e pelas políticas de reclusão das pessoas com deficiência, desenvolveram-se princípios da educação especial, que utilizaram um modelo de déficit, com orientação psicopedagógica para aqueles que defendiam uma educação especializada conduzida por professores especialmente formados em centros especializados. Depois de verificar a segregação e as suas consequências, tem-se avançado com os princípios da normalização e integração. Os alunos com deficiência, necessidades educativas especiais ou necessidades de apoio educativo começaram a ser integrados em ambientes menos restritivos e as escolas começaram a levar em consideração as características e diferenças individuais. São precisamente os princípios da normalização e integração que evoluíram e nos levaram ao princípio da educação inclusiva e do conceito de escola inclusiva ou uma escola para todos. 
Como já mencionado, essa tendência parece ter ocorrido principalmente de forma teórica, mas, felizmente, nos últimos anos também de forma legislativa. No entanto, ainda não se encontra resolvida na sua aplicação prática: embora os principais responsáveis afirmem que a educação inclusiva já existe em nossas escolas, continuamos hoje a confundir os conceitos de integração e inclusão social (Verdugo, 2009). É verdade que ambos os substantivos compartilham muitas semelhanças na semântica, mas na educação em particular e nos setores sociais em geral, não podemos utilizá-los de forma indiscriminada. Embora os objetivos sejam aparentemente semelhantes lou seja, a inclusão das pessoas com deficiência na sociedade), a perspectiva e a filosofia das duas construções são radicalmente diferentes. 0 conceito de inclusão vai além do conceito de integração, ou seja, não se trata de garantir que estudantes com deficiência e sem deficiência compartilhem os mesmos espaços, os mesmos contextos e o mesmo currículo, mas que as escolas os incluam, independentemente do ambiente social, da cultura de origem, da ideologia, sexo, etnia ou situações pessoais resultantes de deficiência física, intelectual, sensorial ou de superdotação intelectual. Para isso, junto com muitas outras ações, é necessário trabalhar as atitudes e os estereótipos, qualificar os professores e professoras assim como outros profissionais envolvidos, mudar a dinâmica da programação educacional e a concepção de currículo habitual e os contextos em que as pessoas são incluídas (Verdugo, 2009).

Deste modo, a diferença da escola inclusiva, como indicado por vários autores (Moriña, 2002; Verdugo, 2009), compreende distintos aspectos: al destina-se não só aos alunos com necessidades especiais que necessitam de apoio educacional, mas aos estudantes em geral; b) concentra-se na resolução de problemas mais que no diagnóstico; cl orienta-se pelos princípios de equidade, cooperação e solidariedade, considerando as diferenças como uma fonte de enriquecimento da sociedade; d) preza pela inclusão total e incondicional dos alunos; el exige uma profunda transformação do sistema educativo e uma ruptura das práticas tradicionais; f) centra-se na sala de aula, proporcionando os apoios necessários ao aluno na sala de aula regular, e não em programas específicos.

Neste ponto, devemos destacar que as mudanças apontadas na atenção educacional até chegar ao princípio da educação inclusiva não aconteceram de forma isolada, mas estiveram estreitamente relacionadas com as mudanças que ocorreram historicamente na própria concepção de deficiência (Crespo; Campo; Verdugo, 2003; Schalock; Verdugo, 2007; Verdugo, 2003), que evoluiu a partir de modelos que consideravam a deficiência como um problema, um transtorno ou déficit aos modelos que consideram a deficiência da pessoa em seu contexto e de acordo com as interações que nele acontecem (Verdugo, 2009). A estes se aproximam elementos fundamentais: o paradigma de apoios individualizados e direcionados para promover mudanças nas variáveis ambientais junto aos 
alunos assim como os modelos de qualidade de vida e autodeterminação, que defendem a atenção personalizada, a avaliação sistemática dos resultados pessoais, as aspirações, opiniões e desejos das pessoas como aspectos imprescindíveis de qualquer intervenção e como elementos essenciais na prática educativa .

\section{Avanços e obstáculos na educação inclusiva na Espanha}

As escolas da Espanha contam hoje com 25 anos de experiência, primeiro integradoras e depois inclusivas. Embora este período tenha sido acompanhado de avaliações sistemáticas dos progressos alcançados e dos obstáculos encontrados ao longo do caminho, ainda hoje persiste a necessidade de se estudar com rigor científico e de forma exaustiva as maneiras pelas quais os principais agentes do processo da educação inclusiva, alunos com deficiência, familiares, profissionais vivenciaram e perceberam tais experiências. Existem revisões abrangentes e boas sínteses sobre a história e a evolução do processo de integração que incluem tanto as percepções dos alunos e suas famílias sobre a educação inclusiva, como as carências e atitudes de professores e professoras. Também encontramos vários estudos que destacam a necessidade de aumentar a presença e a implementação de políticas educacionais destinadas a melhorar e aprofundar o processo de inclusão educacional de alunos com deficiência em grande parte do país (Casanova; Cabra de Luna, 2009; Lorenzo; Perez, 2007; Echeita et al., 2009; Giné, 2006; Ortiz; Lobato, 2003).

As publicações abundantes de especialistas seguem um caminho de postulados inclusivos pautados em princípios e diretrizes comuns, ainda que suas reflexões estejam focadas em diferentes aspectos da inclusão: na filosofia em geral, na inclusão com foco nas necessidades educativas decorrentes da deficiência, na interpretação da mesma, nas metodologias, nos projetos dentro das escolas, na qualidade de vida dos alunos ou na autodeterminação (cf. Ainscow; Booth; Dyson, 2006; Arnaiz; Soto, 2003; Echeita et al., 2009; Stainback, 2001; Verdugo, 2006; Wehmeyer, 2009). Estas contribuições têm se expandido no campo de estudos e de investigação sobre a inclusão que se somam ao crescente número de profissionais da educação, da psicologia ou da sociologia. Contudo, até agora, os estudos têm abordado principalmente questões mais teóricas em detrimento da análise dos dados e evidências das experiências segundo as observações e avaliação de todos os envolvidos, dos quais dependem, em última análise, o sucesso e o fracasso da inclusão. Neste sentido, apresentamos a seguir dois estudos recentes que tiveram lugar no contexto espanhol e que apontam as opiniões e experiências dos principais agentes implicados no processo de implementação da inclusão em contextos educativos. 


\section{Os pontos de vista das organizações no âmbito dos serviços sociais}

Em um estudo realizado por Echeita et al. (2009) buscou-se identificar a visão sobre o processo de educação inclusiva (Ainscow; Booth; Dyson, 2006) por parte de especialistas em matéria de educação das organizações de pessoas com deficiência que faziam parte da comissão de educação da CERMI (Conselho estadual de representantes de pessoas com deficiêncial, utilizando-se para tanto um questionário elaborado ad hoc. Os resultados do estudo revelaram que existiam diferenças significativas na percepção dos 286 participantes da pesquisa la maioria técnicos contratados pelas organizações com mais de oito anos de experiêncial com relação aos processos de aprendizagem e o grau de participação escolar entre os três grupos de alunos com deficiências (visual, auditiva e intelectual), dependendo da etapa escolar que cursavam.

A este respeito, informa-se que entre os pesquisados que se encontravam na fase da educação infantil ( 0 a 6 anos), os níveis de satisfação com o processo de inclusão, especialmente no caso de alunos com deficiência intelectual, foram os mais elevados. Na fase da educação primária (6-12 anos), a maioria das diferenças explicadas pela variável deficiência foram atendidas, visto que é na educação infantil que, aparentemente, existem as melhores condições em termos de educação inclusiva, não existindo, dessa forma, diferenças significativas quanto à aprendizagem e à participação de alunos e alunas com deficiência.

No entanto, essa transição não conduz a êxitos semelhantes nas fases seguintes. Entre os diferentes grupos analisados, foram registradas situações muito desiguais, especialmente para aqueles com deficiência intelectual. Na verdade, os técnicos pesquisados relataram que os alunos e alunas que começaram seus estudos com avaliações elevadas, mostraram durante a escolaridade na educação secundária obrigatória (12-16 anos) os níveis mais baixos de satisfação com relação a aprendizagem e participação, sobretudo em relação ao tratamento recebido e ao nível de autoestima. A pesquisa revelou que os processos de inclusão não têm gerado bons resultados e a participação esperada; pelo menos não em todos os níveis educacionais e nem nos diferentes tipos de deficiência. No entanto, como reconhecem os autores, é imprescindível participar no processo de avaliação dos atores fundamentais, aqueles que até agora foram os menos escutados, os alunos e alunas com deficiência. 


\section{A opinião dos alunos com deficiência, familiares e profissionais}

Com vistas a incluir as opiniões e experiências dos principais atores no processo de educação inclusiva, Rodríguez-Aguilella e Verdugo (2009) realizaram um estudo que procurou analisar a opinião dos alunos com deficiência intelectual, junto aos familiares e aos profissionais da educação. Tratava-se de identificar os aspectos com os quais estavam satisfeitos, os obstáculos enfrentados e as sugestões de melhoria que poderiam facilitar o processo de inclusão em salas de aula. Os autores adotaram uma abordagem qualitativa que envolveu a realização de 18 grupos focais, com seis pessoas em cada grupo (alunos, familiares e profissionais). Os participantes foram escolhidos em seis comunidades autônomas espanholas: Andaluzia, Castilla la Mancha, Castilla y León, Catalunha, Galiza e Madrid. A escolha das regiões participantes foi realizada com base em critérios de representatividade e de disponibilidade de Centros pertencentes à Confederação Espanhola de Organizações para Pessoas com Deficiência Intelectual (FEAPS). Para a análise das informações obtidas foi utilizado o software NUD*DIST, que permite tirar conclusões a partir de uma análise rigorosa do discurso, cujo sistema de indexação está pautado em uma estrutura hierárquica de conceitos na forma de árvore invertida, criando nós, onde cada nó tem um nome e um nível. Na análise, seguimos a proposta de Miles e Huberman (1994): a) redução de dados; b) fornecimento e tratamento de dados; c) obtenção de resultados e verificação das conclusões.

Entre os avanços alcançados na educação inclusiva, os três grupos lalunos, familiares e profissionais) indicaram uma maior satisfação com as conquistas da educação infantil e educação primária. Também apontaram, como já observado no estudo anterior, que na educação secundária aumentam as dificuldades e os problemas. Mencionaram ainda uma maior conscientização entre os professores e professoras, que se reflete em um aumento de iniciativas e projetos inclusivos. Notaram progressos sobretudo na adaptação dos apoios e no envolvimento dos profissionais de educação na etapa da educação primária. Outros problemas e obstáculos foram encontrados e compartilhados no processo da educação inclusiva, por exemplo, desgaste emocional dos pais diante dos contínuos obstáculos encontrados; atitudes negativas; falta de formação de muitos profissionais da educação sobre a deficiência e comportamento de escusa por parte dos professores e professoras; falta de aceitação e comportamentos de abuso ou de maltrato por parte dos próprios colegas da educação secundária obrigatória; falta de habilidades sociais por parte dos estudantes com deficiência; baixo nível de motivação e alto grau de frustração; falta de alternativas para o futuro e problemas de inserção no mercado de trabalho.

Embora os resultados possam parecer um pouco surpreendentes a priori, são reveladores, especialmente se tivermos em conta que, pela primeira vez, levantam-se 
evidências empíricas sobre a situação atual do processo de educação inclusiva na Espanha, em um estudo desta dimensão e escutando o aluno(a) com deficiência intelectual. Entretanto, nos parecem mais importantes as mudanças compartilhadas pelos alunos e alunas com relação à família e aos profissionais da educação. A maior parte dos discursos centrados nas mudanças necessárias para melhorar o processo de inclusão educacional teve como foco a melhoria das atitudes em relação à deficiência e à formação especializada de professores. Outra questão fundamental para o sucesso da educação inclusiva, diz respeito as relações professor-aluno em sala de aula, que devem ter como base a diversidade e as necessidades específicas de cada grupo. Também foi essencial a coordenação entre os vários sistemas envolvidos (incluindo, entre outros, professores, famílias e discentes), que poderia ser melhorada simplesmente organizando-se reuniões com mais frequência. Finalmente, no âmbito legislativo, foram propostos como uma necessidade urgente o desenvolvimento e a implementação de alternativas para o prosseguimento dos estudos, assim como a conveniência de incluir uma maior quantidade de conteúdos de orientação para a prática nos currículos acadêmicos.

\section{Avanços no processo de educação inclusiva}

Apesar dos resultados apresentados nas pesquisas citadas acima, houve uma certa estagnação nos últimos anos, e até mesmo retrocesso no progresso escolar para atender às pessoas com deficiência e outras limitações, com uma grande apatia institucional, profissional e social para promover a inclusão educacional. No entanto, a atual situação é novamente positiva e esperançosa para favorecer a tomada de decisões promotoras de práticas generalizadas e inclusivas nas escolas. Longe de nos situarmos em posições negativistas e pessimistas que dificultam a ação, devemos reconhecer os avanços até o momento, ser otimistas e trabalhar em uma direção comum: a realização de uma verdadeira educação com inclusão educacional concreta e eficiente. Portanto, devemos ressaltar os progressos realizados no governo espanhol e algumas das administrações autônomas (regionais) na área da educação; a progressiva e crescente responsabilidade em busca da melhoria do processo inclusivo; a intensificação dos esforços para convidar ao diálogo e ouvir a voz dos profissionais, acadêmicos/as, professores e professoras, pessoal de atendimento direto, familiares, pessoas com deficiência, assim como todos os envolvidos e interessados no desenvolvimento de práticas inclusivas. Finalmente, muitas organizações sociais representativas de grupos com necessidades de apoio e com risco de exclusão social têm manifestado ativamente sua demanda por mais e 
melhor compromisso para a inclusão educacional de alunos com deficiência e outras necessidades especiais de apoio educativo.

Embora ainda haja muito a ser feito para se alcançar uma educação plenamente inclusiva, o estudo mostra que houve progressos significativos, que não devem ser ignorados, mas com os quais tampouco devemos nos acomodar. Esses avanços são necessários mas insuficientes. São apenas os primeiros passos em direção ao que deveria ser a meta de todos os envolvidos e interessados nos processos e contextos. Estamos agora no momento certo para ir além da preocupação e da autoconsciência da situação atual da educação do aluno ou aluna com deficiência; devemos começar a planejar de forma sistemática e coordenada as iniciativas, atuações, avaliações e intervenções educativas.

Devemos buscar mecanismos que complementem a educação inclusiva com a criação de condições para que os direitos reconhecidos nas legislações vigentes sejam reais e efetivos. No contexto de uma escola inclusiva que reconheça os direitos de todos os alunos a participar nos ambientes naturais, devemos especificar a direção dos esforços e critérios para avaliar os progressos alcançados. Nesse sentido, uma abordagem multidimensional para o aluno ou aluna focada em qualidade de vida (Schalock; Gardner; Bradley, 2007; Schalock; Verdugo, 2002; 2006; 2007; 2009) poderia ajudar a estruturar o planejamento dos processos de ensino e aprendizagem. Para agilizar e sistematizar a implementação das práticas, intervenções e avaliações educacionais é imprescindível a utilização do desenho universal comum de ensino-aprendizagem e do currículo escolar (Wehmeyer, 2009) e de uma abordagem multidimensional dos alunos e alunas com e sem deficiência, centrada na sua qualidade de vida.

\section{0 modelo de vida como um agente de mudança em contextos escolares}

\section{Educação inclusiva e qualidade de vida}

Como mostrado anteriormente, um dos grandes desafios do sistema educacional é garantir que as escolas inclusivas sejam uma realidade e que verdadeiramente ofereçam respostas de qualidade a todos os alunos. Trata-se de alcançar igualdade de oportunidade, participação e excelência, de tal forma que o aluno ou aluna consiga

desenvolver plenamente (ao máximo) suas potencialidades e que a escola possa aceitar as diferenças individuais, proporcionando apoios individualizados e atenção à diversidade centrada na pessoa (Schalock; Verdugo, 2002). 
O paradigma da qualidade de vida apoia a educação inclusiva e permite avançar em direção a uma "educação integral", levando em consideração todas as dimensões da vida do estudante. Mais concretamente, o modelo de qualidade de vida serve como referência, como marco conceitual para mudanças curriculares e outras transformações que os contextos educacionais devem realizar para atender as necessidades dos alunos, considerando suas opiniões e preferências, fornecendo-lhes os apoios individualizados que necessitam. A inclusão do modelo de qualidade de vida nas avaliações e intervenções educativas permitem melhorar o planejamento educacional, desenvolver modelos de avaliação de programas centrados na pessoa, aumentar a participação dos usuários nos processos e decisões que lhes dizem respeito, avaliar os efeitos da educação inclusiva nas áreas acadêmica e não acadêmica, assim como orientar as intervenções destinadas a aumentar a satisfação dos usuários e a participação ativa dos alunos com e sem deficiência. A adoção de um modelo de qualidade de vida em um contexto educacional inclusivo envolve profundas mudanças sociais e a participação e interação do sistema familiar e da comunidade, que vão além do mero "estar em um ambiente inclusivo", proporcionando oportunidades e experiências no contexto da comunidade próxima, que terá impacto sobre a qualidade de vida dos alunos.

Apesar do modelo de qualidade de vida ser essencial em todas as etapas educativas, incluindo a educação infantil e intervenção precoce (pois pode dirigir e orientar grande parte das avaliações e intervenções realizadas), o conceito é particularmente importante a partir da educação secundária obrigatória, uma vez que a análise das dimensões e indicadores de qualidade de vida não só são importantes na educação, mas essenciais também para a autonomia, emprego e oportunidades de tomada de decisões que são esperadas na vida adulta. Entre ambas as fases, não devemos esquecer a etapa de transição para a vida adulta, na qual a oferta de apoios especializados e atenção personalizada é de vital importância e na qual o modelo de qualidade de vida dos indivíduos e das famílias deve se tornar novamente o principal impulsionador das práticas de avaliação e intervenção. A chave para o sucesso de modelos de qualidade de vida no esforço para conseguir uma verdadeira educação inclusiva se encontra na necessidade de levar em consideração não apenas as variáveis diretamente relacionadas ao sucesso escolar e ao processo de ensino-aprendizagem, mas também os múltiplos aspectos da vida que são importantes para alunos e alunas com e sem deficiência.

Deste modo, o conceito de qualidade de vida se apresenta como uma abordagem holística e multidimensional, com foco na pessoa e que ajuda a especificar os indicadores mais importantes de qualidade de vida (Schalock; Verdugo, 2002; 2007; Schalock et al., 2008; Verdugo, 2006). Ao contrário de outros conceitos mais globais, falar de uma abordagem pautada na qualidade de vida de alunos e alunas implica na avaliação de resultados 
pessoais como critério para identificar necessidades e definir programas, ao mesmo tempo em que implica constatar os avanços que foram feitos no processo de integração e planejamento dos apoios individuais.

\section{Conceituação da qualidade de vida: o modelo de oito dimensões}

Entre os diversos modelos de qualidade de vida centrados na pessoa, o modelo proposto por Schalock e Verdugo $(2002 ; 2007)$ destaca-se pela sua crescente utilização e pelo número de citações no campo da deficiência. Embora o modelo de Schalock e Verdugo tenham sido utilizado principalmente na área da deficiência intelectual (Verdugo et al., 2009; Verdugo; Gomez; Arias, 2007; Verdugo et al., 2010a), seu uso está se estendendo atualmente a outros grupos, por exemplo: pessoas com deficiência visual (Verdugo et al., 2005), idosos (Gomez et al., 2008), pessoas com dependência de drogas (Arias et al., 2010), pessoas com lesão medular (Aguado; Alcedo, 2005), usuários dos serviços sociais em geral e pessoas sem deficiência (Gomez-Vela, 2007). Além disso, sua aplicação se estende por todo ciclo da vida e orienta as práticas na estimulação precoce, na educação infantil, no ensino fundamental e médio e nos programas de transição para a idade adulta, na vida adulta e na velhice.

0 modelo se operacionaliza através de dimensões, indicadores e resultados pessoais que são organizados em diferentes níveis (Gomez; Verdugo; Arias et al. 2010). As dimensões de qualidade de vida propostas pelo modelo são oito: bem-estar emocional, relações interpessoais, bem-estar material, desenvolvimento pessoal, bem-estar físico, autodeterminação, inclusão social e direitos. As dimensões básicas de qualidade de vida são entendidas como um conjunto de fatores que compõem o bem-estar pessoal (Schalock; Verdugo, 2002). As dimensões de qualidade de vida são operacionalizadas mediante seus indicadores centrais (Schalock; Gardner; Bradley, 2007; Schalock et al., 2010; Verdugo et al., 2010b), que se definem como percepções, comportamentos ou condições específicas das várias dimensões da qualidade de vida que refletem o bemestar de uma pessoa (Schalock; Verdugo, 2002). A avaliação da situação e as aspirações pessoais nestes indicadores refletem sobre o desempenho pessoal, que se configura como aspirações definidas e valorizadas pelas pessoas (Schalock; Gardner; Bradley, 2007).

Deste modo, no modelo de qualidade de vida entende-se que a medida dos indicadores está sempre relacionada a resultados pessoais da qualidade de vida. Com base em evidências e não em meras suposições ou interpretações pessoais, propõe-se a seguinte definição operacional de qualidade de vida referente à pessoa: 
Qualidade de vida individual é um fenômeno multidimensional, composto de dimensões principais que são influenciadas por características pessoais e fatores ambientais. Estas dimensões principais são iguais para todas as pessoas, mas podem variar em seu valor relativo e importância. As dimensões da qualidade de vida são avaliadas por meio de indicadores que são sensíveis à cultura (Schalock et al., 2010, p.21).

\section{Avaliação da qualidade de vida em contextos educativos}

0 modelo de qualidade de vida (Schalock; Verdugo, 2002; 2007), por conter várias evidências empíricas sobre sua conceituação, formulação, implementação e validação em vários países, tanto ocidentais como orientais, torna-se um valioso instrumento para orientar e avaliar o progresso nas mudanças da escola de acordo com a constatação dos resultados alcançados, centrados na pessoa. 0 interesse crescente na avaliação da qualidade de vida dos alunos com necessidades específicas faz parte de uma tendência cultural mais ampla de interesse para avaliar e documentar os dados dos avanços e conquistas na educação que envolvem instituições acadêmicas, organizações não governamentais (ONGs) e serviços sociais.

A pesquisa sobre qualidade de vida tem demonstrado grande atividade na Espanha nos últimos anos. Os estudos estão revelando progressos significativos que se concentram principalmente no desenvolvimento de instrumentos e sua validação tanto no âmbito educativo geral (Gomez-Vela; Verdugo, 2009; Sabeh et al., 2009), como na vida adulta das pessoas com deficiência intelectual (Verdugo; Arias; Gomez, 2006; Verdugo et al., 2009; 2010; Verdugo; Gomez; Arias, 2007) ou nos serviços sociais para os diferentes grupos (Verdugo et al., 2007; 2009; 2010b). Além disso, estudamos também a qualidade da vida familiar com a validação de instrumentos adequados (Verdugo; Córdoba; Gomez, 2005) e em relação à adaptação e à satisfação do sistema familiar em diferentes situações, como as crianças com hiperatividade ou pessoas em fase de envelhecimento com deficiência (Rodríguez-Aguilella; Verdugo; Sánchez-Gomez, 2008). No entanto, a maioria dos estudos está focada principalmente na população adulta, são escassos os estudos realizados com pessoas com deficiência em idade escolar.

Ainda assim, o conceito de qualidade de vida tem sido explicitamente proposto nos últimos anos como um marco de referência para definir os objetivos no âmbito da educação especial e inclusiva para alunos e alunas com necessidades educacionais especiais (Schalock; Verdugo, 2002; Turnbull et al., 2006). Mas são poucos os profissionais da educação que incluem estas dimensões - relações interpessoais, bem-estar emocional, autodeterminação -, em suas práticas. Nesse sentido, convém considerar o uso de novos instrumentos que permitam a avaliação da qualidade de vida em vários contextos. 


\section{Considerações finais}

Apesar dos progressos na incorporação de alunos e alunas com deficiência nas escolas regulares nas últimas décadas, a educação de pessoas com necessidades específicas de apoio educativo na Espanha tem passado por momentos difíceis, que indicam a necessidade de estar constantemente em alerta para promover ações que favoreçam os avanços sem cair em retrocessos. Esses problemas que já existiam, e em certa medida ainda existem, nos indicam as reclamações e demandas dos alunos e alunas com deficiência, suas famílias, os professores e professoras e profissionais que trabalham com eles, juntamente com organizações que defendem seus direitos e procuram proporcionar-lhes os apoios adequados (Echeita et al., 2009). Revelam-nos obstáculos encontrados no processo de implementação da educação inclusiva e necessidade urgente de um maior envolvimento das instituições públicas e um papel mais ativo e coordenado de todos os agentes implicados.

A perspectiva de qualidade de vida converte-se em um aspecto essencial do processo de mudança educacional, ao oferecer um modelo que permite planejar individualmente os apoios dos alunos e alunas, dentro de uma perspectiva integral lacadêmica e social, incluindo os seus desejos, objetivos e preferências), assim como avaliar de forma sistemática os progressos alcançados. 0 modelo de qualidade de vida ressalta a necessidade de ir além das conquistas puramente acadêmicas e incluir outros aspectos importantes para todas as pessoas, como parte do currículo. Somente por meio de uma avaliação sistemática, utilizando instrumentos com evidências adequadas de validade e confiabilidade, pode-se planejar e implementar práticas educativas e profissionais orientadas a alcançar melhorias constatáveis na educação integral dos alunos. Além disso, outros aspectos determinantes são a melhoria e atualização constante dos profissionais, o envolvimento dos alunos com deficiência e suas famílias, o planejamento e implementação de intervenções baseadas em evidências, a provisão de apoios para melhorar a qualidade de vida e a autodeterminação, a promoção de práticas educativas inovadoras e inclusivas, a supervisão dos processos para verificar os resultados e identificar os obstáculos a superar, e a melhoria na detecção precoce das deficiências e das necessidades específicas de apoios educacionais.

Deste modo, defende-se a integração da perspectiva de qualidade de vida, autodeterminação e apoios ao longo de todo o ciclo de vida, desde a educação infantil ou de intervenção precoce, passando pela educação primária e especialmente a educação secundária, até a etapa de transição para a vida adulta com alcance da integração no mercado de trabalho. A mesma perspectiva deverá continuar na idade adulta e na velhice. 
Finalmente, recomendamos o uso de uma estratégia sistemática, com diferentes táticas que ampliem as possibilidades e a eficácia das propostas de mudança. Nesse sentido, é preciso implementar ações nos diversos sistemas envolvidos na atividade educativa: o microsistema, mediante mudanças e inovações nas práticas educativas e inclusivas; o mesosistema, por meio de mudanças nas organizações que oferecem apoios nos diferentes sistemas e contextos educativos; o macro-sistema, através de mudanças na política da educação. Nesse sentido, o modelo de qualidade de vida (Schalock; Verdugo, 2002) serve de referência geral para que tais mudanças sejam promovidas e mantidas.

\section{Referências}

AGUADO, Antonio; ALCEDO, María Ángeles. Una visión psicosocial del envejecimiento de las personas con lesión medular. Intervención Psicosocial, Madrid, v. 14, n. 1, p. 51-63, 2005.

AINSCOW, Mel; BOOTH, Tony; DYSON, Alan. Improving schools, developing inclusion. Londres: Routledge, 2006.

ARIAS, Benito et al. Evaluación de la calidad de vida en personas drogodependientes mediante el modelo de Rasch. Revista Española de Drogodependencias, Valencia, v. 35, n. 2, p. 206-219. 2010.

ARNÁlZ, Pilar; SOTO, Gloria. Special Education in Spain at the beginning of the 21st century: the challenge to educate in an unequal and multicultural society. International Journal of Inclusive Education, Londres, v. 7, n. 4, p. 375-388, 2003.

CASANOVA, Maria Antonia; CABRA DE LUNA, Miguel Ángel. Educación y personas con discapacidad: presente y futuro. Madrid: Fundación Once, 2009.

CRESPO, Manuela; CAMPO, Maribel; VERDUGO, Miguel Ángel. Historia de la clasificación internacional del funcionamiento de la discapacidad y de la salud (CIF): un largo camino recorrido. Siglo Cero, Madrid, v. 34, n. 1, p. 20-26, 2003.

ECHEÍTA, Gerardo et al. Paradojas y dilemas en el proceso de inclusión educativa en España. Revista de Educación, Madrid, n. 349, p. 153-178, 2009.

GINÉ, Climent. Reflexions sobre l'educació dels alumnes amb necessitats educatives especials: situació actual i perspectives de futur. Número Monográfico. Suports Revista Catalana d'Educació Inclusiva, Cataluña, v. 10, n. 1, p. 21-24, 2006.

GÓMEZ-VELA, María. La calidad de vida de alumnos con necesidades educativas especiales y sin ellas. Elaboración de un marco conceptual y un instrumento de evaluación. Educación y Diversidad, Zaragoza, v. 1, p. 113-135, 2007.

GÓMEZ-VELA, María; VERDUGO, Miguel Ángel. Cuestionario de evaluación de la calidad de vida en alumnos adolescentes (CCVA). Madrid: CEPE, 2009.

GÓMEZ, Laura Elísabet et al. Evaluación de la calidad de vida en personas mayores y con discapacidad: la Escala FUMAT. Intervención Psicosocial, Madrid, v. 17, n. 2, p. 189-200, 2008. 
. VERDUGO, Miguel Ángel; ARIAS, Benito. El concepto de calidad de vida individual: avances en su conceptualización y retos emergentes. Psicología Conductual, Granada, v. 18, n. 3, p. 553-472, 2010. LORENZO, Rafael; PÉREZ, Luis. Tratado sobre Discapacidad. Madrid: Aranzadi/Thomson, 2007.

MILES, Matthew; HUBERMAN, Michel. Qualitative data analysis: an expanded sourcebook. Londres: Sage, 1994.

MORIÑA, Anabel. El camino hacia la inclusión en España: una revisión de las estadísticas de Educación Especial. Revista de Educación, Madrid, v. 327, p. 395-414, 2002.

ORTIZ, María del Carmen; LOBATO, Xilda. Escuela inclusiva y cultura escolar: algunas evidencias empíricas. Bordón, Revista de Pedagogía, Madrid, v. 55, n. 1, p. 27-40, 2003.

RODRÍGUEZ-AGUILELLA, Alba; VERDUGO, Miguel Ángel; SÁNCHEZ-GÓMEZ; Maria Cruz. Calidad de vida familiar y apoyos para los progenitores de personas con discapacidad intelectual en proceso de envejecimiento. Siglo Cero, Madrid, v. 39, n. 3, p. 19-34, 2008.

RODRÍGUEZ-AGUILELLA, Alba; VERDUGO, Miguel Ángel. Valoración de La inclusión educativa desde diferentes perspectivas. Siglo cero, Madrid, v.39, n. 4, p.5-25, 2009.

SABEH, Eliana et al. CVI-CVIP Cuestionarios de evaluación de la calidad de vida en la infancia. Madrid: CEPE, 2009.

SCHALOCK, Robert; GARDNER; James; BRADLEY, Valerie. Quality of life for persons with intellectual and other developmental disabilities: Applications across individuals, organizations, communities, and systems. Washington, DC: American, 2007.

. VERDUGO, Miguel Ángel. The concept of quality of life in human services: A handbook for human service practitioners. Washington, DC: American Association on Mental Retardation. 2002.

. VERDUGO, Miguel Ángel. El concepto de calidad de vida en los servicios y apoyos para personas con discapacidad intelectual. Siglo Cero, Madrid, v. 38, n. 4, p. 21-36, 2007.

, et al. Enhancing personal outcomes: organizational strategies, guidelines, and examples. Journal of Policy and Practice in Intellectual Disabilities, v. 5, n. 1, p. 18-28, 2008.

STAINBACK, Susan. Aulas inclusivas: un nuevo modo de enfocar y vivir el curriculum. Madrid: Narcea, 2001.

TURNBULL, Rutherford et al. A Quality of Life Framework for Special Education Outcomes. Remedial and Special Education, v. 24, n. 2, p. 67-74, 2006.

VERDUGO, Miguel Ángel. Análisis de la definición de discapacidad intelectual de la Asociación Americana sobre Retraso Mental de 2002. Siglo Cero, Madrid, v. 34, n. 1, p. 5-19, 2003.

. Como mejorar la calidad de vida de las personas con discapacidad. Salamanca: Amarú. 2006.

El cambio educativo desde una perspectiva de calidad de vida. Monográfico. Revista de Educación, Madrid, n. 349, p 23-43. 2009.

. CÓRDOBA, Leonor; GÓMEZ, Juan. The Spanish Adaptation and Validation of the Family Quality of Life Survey. Journal of Intellectual Disability Research, v. 49, p. 492-498, 2005.

et al. Factorial structure of the quality of life questionnaire in a Spanish sample of visually disabled adults. European Journal of Psychological Assessment, v. 21, n. 1, p. 44-55, 2005. 
. ARIAS, Benito; GÓMEZ, Laura Elísabet. Escala integral de medición subjetiva y objetiva de la calidad de vida en personas con discapacidad intelectual. In: Como mejorar la calidad de vida de las personas con discapacidad. Instrumentos y estrategias de evaluación. Salamanca: Amarú, 2006, p. 417-448.

GÓMEZ, Laura Elísabet; ARIAS, Benito. La Escala Integral de Calidad de Vida. Desarrollo y estudio preliminar de sus propiedades psicométricas. Siglo Cero, Madrid, v. 38, n. 4, p. 37-56, 2007. . et al. Escala Integral. Evaluación objetiva y subjetiva de la calidad de vida de las personas con discapacidad intelectual. Madrid: CEPE, 2009.

. et al. The Integral quality of life scale: development, validation, and use. In: Enhancing the Quality of Life of People with Intellectual Disabilities: from Theory to Practice. Londres: Springer, 2010a, p. 47-60.

. et al. Development of an objective instrument to assess quality of life in social services: Reliability and validity in Spain. International Journal of Clinical and Health Psychology, Granada, v. 10, n. 1., p. 105-123, 2010b.

WEHMEYER, Michael. Autodeterminación y la tercera generación de prácticas de inclusión. Revista de Educación, Madrid, n. 349, p. 45-67. 2009.

Recebido em fevereiro de 2011

Aprovado em junho de 2011

Miguel Ángel Verdugo Alonso, doutor em Psicologia, catedrático de Psicologia da Deficiência, diretor do Instituto Universitário de Integração na Comunidade, do Serviço de Informação sobre Deficiência (SID) e do mestrado em Qualidade de Vida e Inclusão de Pessoas com Deficiência na Universidade de Salamanca. Diretor da revista científica “Siglo Cero". Publicação recente: Adaptation and psychometric properties of the Spanish version of the Supports Intensity Scale SIS ( American Journal of Intellectual and Developmental Disabilities, 115 (6), 496-503, 2010) em co-autoria com Arias, B., Ibáñez, A.; Schalock, R. L. E-mail: verdugodusal.es.

Laura Elisabet Gómez Sánchez, doutora em Psicologia e professora ajudante doutora no Departamento de Psicologia da Universidade de Oviedo (Espanha). Publicação recente: A comparison of alternative models of individual quality of life for social service recipients (Social Indicators Research, 2011). E-mail: lauragomezdpsi.uv.es.

Alba Rodriguez Aguilell, doutora em Psicologia. Membro do Instituto Universitário de Integração na Comunidade - UNICO. Publicação recente: La inclusión educativa en España desde la perspectiva de alumnos con discapacidad intelectual, de familias y de profesionales (Revista Educación, 2010, DOI: 10-4438/1988-592x-RE-2010-358-086). E-mail: albarodriguezQusal.es 